\title{
PROOF OF A THEOREM OF SAKS AND SIERPINSKI
}

\section{CASPER GOFFMAN}

The object of this note is to give a direct proof of the following theorem of Saks and Sierpinski:

If $f(x)$ is an arbitrary one-valued real function defined on the closed interval $I=[0,1]$, there is a $\phi(x)$ of Baire class 2 at most such that for every $\epsilon>0$ the inequality $|f(x)-\phi(x)|<\epsilon$ holds on a set of exterior measure 1.

The proofs in the literature $[1,2]^{1}$ depend on the corresponding theorem for measurable functions; namely, if $f(x)$ is measurable, there is a $\phi(x)$ of Baire class 2 at most such that $f(x)=\phi(x)$ almost everywhere.

We first prove a lemma which seems to be new.

LEMMA. If $f(x)$ is defined on the closed interval $I=[0,1], \epsilon>0, \phi(x)$ continuous, and $|f(x)-\phi(x)|<\epsilon$ on a set of exterior measure greate ${ }^{r}$ than $1-\epsilon$, then for every $\eta>0$ there is a continuous $\psi(x)$ such tha $|\phi(x)-\psi(x)|<\epsilon$ on a set of measure greater than $1-\epsilon$ and $|f(x)-\psi(x)|$ $<\eta$ on a set of exterior measure greater than $1-\eta$.

PROof. $f(x)$ is exteriorly approximately continuous almost everywhere in the sense that for almost every $\xi \in I$ the set of points $x$ for which $f(\xi)-k<f(x)<f(\xi)+k$ has exterior metric density 1 at $\xi$, for every $k>0$. There is a $\delta$, with $0<\delta<\eta$, such that $|f(x)-\phi(x)|<\epsilon-\delta$ on a set $E$ of exterior measure greater than $1-\epsilon$. Since $f(x)$ is exteriorly approximately con tinuous almost everywhere, every $\xi \in E-Z$, with $Z$ of measure zero, is in a sequence, $\left\{I_{\xi_{n}}\right\}, i=1,2, \cdots$, of closed intervals, whose lengths converge to zero, such that the set of points $x$ for which $\mid f(\xi)-f(x)<\delta$ has relative exterior measure exceeding $1-\delta / 2$ in each $I_{\xi n}$. Moreover, since $\phi(x)$ is continuous, the $I_{\xi n}$ may be chosen so that the saltus of $\phi(x)$ in $I_{\xi n}$ is less than $\delta$ for every $n$. Consider the totality of intervals

$$
I=\left[I_{\xi_{n}}\right], \quad \xi \in E-Z, n=1,2, \cdots .
$$

By Vitali's covering theorem, since $m_{e}(E-Z)>1-\epsilon$, there is a finite number $I_{\xi_{1} n_{1}}, I_{\xi_{2} n_{2}}, \cdots, I_{\xi_{k} n_{k}}$ of disjoint intervals of $I$ the sum, $\sum_{i=1}^{k} m\left(I_{\xi_{i} n_{i}}\right)$, of whose lengths is $1-\alpha>1-\epsilon$. Let $G=I-\sum_{i=1}^{k} I_{\xi_{i} n_{i}}$ and let $G^{\prime} \subset G$ be the points of $G$ at which $f(x)$ is exteriorly approxi-

Presented to the Society, December 29, 1947; received by the editors August 20, 1947, and, in revised form, November 19, 1947.

${ }^{1}$ Numbers in brackets refer to the references cited at the end of the paper. 
mately continuous. Every $\zeta \in G^{\prime}$ is in a sequence $\left\{I_{\zeta m}\right\}$ of closed intervals contained in $G$, whose lengths converge to zero, such that for every $m$, the set of points $x$ for which $|f(\zeta)-f(x)|<\delta$ has relative exterior measure in $I_{\zeta m}$ exceeding $1-\delta / 2$. Consider the totality of intervals

$$
J=\left[I_{\zeta m}\right], \quad \zeta \in G^{\prime}, m=1,2, \cdots .
$$

There is then a finite number of disjoint intervals $I_{\zeta_{1^{m}}}, I_{\zeta_{2} m_{2}}, \cdots$, $I_{\zeta_{K^{K}} m_{K}}$ of $J$ such that $\sum_{i=1}^{K} m\left(I_{\zeta_{i} m_{i}}\right)$ exceeds $\alpha-\delta / 2$. Now, let

$$
\begin{array}{ll}
\psi(x)=f\left(\xi_{i}\right) & \text { for every } x \in I_{\xi_{i_{i}}}, i=1,2, \cdots, k, \\
\psi(x)=f\left(\zeta_{i}\right) & \text { for every } x \in I_{\zeta_{i} m_{i}}, i=1,2, \cdots, K,
\end{array}
$$

and let $\psi(x)$ be defined elsewhere so as to be continuous. Suppose $x \in I_{\xi_{i} n_{i}}, 1 \leqq i \leqq k$.

$$
\begin{aligned}
|\psi(x)-\phi(x)| \leqq & \left|\psi(x)-f\left(\xi_{i}\right)\right|+\left|f\left(\xi_{i}\right)-\phi\left(\xi_{i}\right)\right| \\
& +\left|\phi\left(\xi_{i}\right)-\phi(x)\right|<0+(\epsilon-\delta)+\delta=\epsilon_{0}
\end{aligned}
$$

But $\sum_{i=1}^{\boldsymbol{k}} m\left(I_{\xi_{i} n_{i}}\right)>1-\epsilon$. Hence $|\psi(x)-\phi(x)|<\epsilon$ on a set of measure greater than $1-\epsilon$. Moreover, since the relative exterior measure of the set of points for which $|\psi(x)-f(x)|<\delta<\eta$ exceeds $1-\delta / 2$ in every $I_{\xi_{i} n_{i}}, i=1,2, \cdots, k$, and in every $I_{\zeta_{i} m_{i}}, i=1,2, \cdots, K$, and these intervals are disjoint, the exterior measure of this set exceeds

$$
\begin{aligned}
\left(1-\frac{\delta}{2}\right)\left[\sum_{i=1}^{k} m\left(I_{\xi_{i n} n_{i}}\right)+\sum_{i=1}^{K}\right. & \left.m\left(I_{\zeta_{i} m_{i}}\right)\right] \\
\geqq & \left(1-\frac{\delta}{2}\right)\left[(1-\alpha)+\left(\alpha-\frac{\delta}{2}\right)\right] \\
& =\left(1-\frac{\delta}{2}\right)^{2}>1-\delta>1-\eta .
\end{aligned}
$$

This completes the proof.

We now prove the theorem of Saks and Sierpinski. Let $f(x)$ be an arbitrary function defined on $I=[0,1]$. Let $\phi_{0}(x) \equiv 0$. By the lemma, there is a continuous $\phi_{1}(x)$ such that $\left|\phi_{1}(x)-f(x)\right|<1 / 2$ on a set of exterior measure greater than $1 / 2$. Having defined the continuous functions

$$
\phi_{1}(x), \phi_{2}(x), \cdots, \phi_{n-1}(x)
$$

with $\left|\phi_{n-1}(x)-f(x)\right|<1 / 2^{n-1}$ on a set of exterior measure greater than $1-1 / 2^{n-1}$ there is, by the lemma, a continuous $\phi_{n}(x)$ such that $\left|\phi_{n}(x)-\phi_{n-1}(x)\right|<1 / 2^{n-1}$ on a set of measure greater than $1-1 / 2^{n-1}$ 
and $\left|\phi_{n}(x)-f(x)\right|<1 / 2^{n}$ on a set of exterior measure greater than $1-1 / 2^{n}$. Let

$$
\phi(x)=\limsup _{n \rightarrow \infty} \phi_{n}(x)
$$

Then $\phi(x)=\lim _{n \rightarrow \infty}\left[\right.$ l.u.b. $\left.\left(\phi_{n}(x), \phi_{n+1}(x), \cdots\right)\right]$, and, since 1.u.b. $\left(\phi_{n}(x), \phi_{n+1}(x), \cdots\right)$ is lower semi-continuous, for every $n, \phi(x)$ is the limit of a nonincreasing sequence of lower semi-continuous functions and is, therefore, of Baire class 2 at most. Moreover, the sequence $\left\{\phi_{n}(x)\right\}$ itself converges almost everywhere to $\phi(x)$. Accordingly, for every $\epsilon>0$ and every $\eta>0$, there is an $n$, such that $\left|\phi_{n}(x)-\phi(x)\right|<\epsilon / 2$ on a set of measure greater than $1-\eta / 2$ and $\left|\phi_{n}(x)-f(x)\right|<\epsilon / 2$ on a set of exterior measure greater than $1-\eta / 2$. Hence, the set of points for which $|\phi(x)-f(x)|<\epsilon$ is of exterior measure greater than $1-\eta$ for every $\eta>0$ and is, therefore, of exterior measure one.

\section{REFERENCES}

1. S. Saks and W. Sierpinski, Sur une propriêté gênérale de fonctions, Fund. Math. vol. 11 (1928) pp. 105-112.

2. H. Blumberg, The measurable boundaries of an arbitrary function, Acta Math. vol. 65 (1935) p. 277.

UNIVERSITY OF OKLAHOMA 